\title{
Das Lymphödemrisiko von Mammakarzinompatientinnen nach Operation oder Strahlentherapie der Axilla
}

\author{
Marciana-Nona Duma ${ }^{1}$ \\ Angenommen: 2. März 2021 / Online publiziert: 25. März 2021 \\ (c) Der/die Autor(en) 2021
}

Einleitung Ziel dieser Studie war es, das Auftreten eines axillären Lymphödems bei Brustkrebspatientinnen (,,breast cancer-related lymphedema“" [BCRL]) in Abhängigkeit von der axillären Operationsart und der regionalen Lymphknotenbestrahlung (,regional lymphnode radiation“ [RLNR]) $\mathrm{zu}$ vergleichen.

Patienten und Methode Von 2005 bis 2018 wurden 1815 Patientinnen mit invasivem Brustkrebs in eine Lymphödemscreeningstudie eingeschlossen. Die Patientinnen wurden in Abhängigkeit von der Art der axillären Behandlung in die folgenden 4 Gruppen eingeteilt: Sentinel-Lymphknoten-Biopsie (SLNB) allein, SLNB+RLNR, axilläre Lymphknotendissektion (ALND) allein und ALND+RLNR. Zur objektiven Beurteilung des Armvolumens wurde ein Perometer verwendet. Bei allen Patienten wurden präoperative Basislinienmessungen und Nachmessungen nach der Behandlung durchgeführt. Ein Lymphödem wurde definiert als eine $\geq 10 \%$ ige relative Zunahme des Armvolumens, welche $>3$ Monate postoperativ auftrat. Der primäre Endpunkt war die BCRL-Rate in den Gruppen. Sekundäre Endpunkte waren die 5-Jahres-Lokalkontrolle und das krankheitsfreie Überleben (DFS).

Ergebnisse Die Kohorte umfasste 1340 Patienten mit SLNB allein, 121 mit SLNB+RLNR, 91 mit ALND allein und 263 mit ALND+RLNR. Die gesamte mediane Nach-

Originalpublikation Naoum GE, Roberts CH, Brunelle CL, Shui AM et al (2020) Quantifying the Impact of Axillary Surgery and Nodal Irradiation on Breast Cancer-Related Lymphedema and Local Tumor Control: Long-Term Results From a Prospective Screening Trial. J Clin Oncol Oct. 10:38(29):3430-3438. https:// doi.org/10.1200/JCO.20.00459. Epub 2020 Jul 30.

\footnotetext{
PD Dr. med. Marciana-Nona Duma

Marciana-Nona.Duma@med.uni-jena.de

1 Klinik für Strahlentherapie und Radioonkologie, Universitätsklinikum Jena, Bachstr. 18, 07745 Jena, Deutschland
}

beobachtungszeit nach der Diagnose betrug 52,7 Monate. Die kumulativen 5-Jahres-Inzidenzraten für BCRL betrugen $30,1 \%, 24,9 \%, 10,7 \%$ bzw. 8,0\% für ALND+RLNR, ALND allein, SLNB+RLNR und SLNB allein. Multivariate Cox-Modelle, die für Alter, Body-Mass-Index, Operation und Rekonstruktionstyp adjustiert waren, zeigten, dass die ALND-allein-Gruppe ein signifikant höheres BCRLRisiko hatte (Hazard Ratio [HR] 2,66; $P=0,02$ ) im Vergleich zur SLNB+RLNR-Gruppe. Es gab keinen signifikanten Unterschied im BCRL-Risiko zwischen den Gruppen ALND+RLNR und ALND allein (HR 1,20; $P=0,49$ ) und zwischen den Gruppen SLNB allein und SLNB+RLNR (HR 1,33; $P=0,44)$. Die lokalen 5-Jahres-Kontrollraten waren in den Gruppen ALND+RLNR, ALND allein, SLNB+RLNR und SLNB allein ähnlich $(2,8 \%, 3,8 \%$, $0 \%$ bzw. $2,3 \%)$.

Schlussfolgerung der Autoren Obwohl die RLNR das Risiko für ein Lymphödem des Arms auch erhöht, ist der Hauptrisikofaktor für das Auftreten eines Lymphödems die Radikalität der axillären Operation (ALND).

\section{Kommentar}

Die Bestrahlung der Lymphabflusswege ist ein entscheidender Bestandteil der adjuvanten Therapie von Mammakarzinompatientinnen mit Lymphknotenbefall. Daten aus der MA.20- und der EORTC-22922-10925-Studie haben bewiesen, dass die zusätzliche Bestrahlung der supraklavikulären und der Mammaria-interna-Lymphabflusswege im Vergleich zur alleinigen Ganzbrust- oder Brustwandbestrahlung die regionale und Fernkontrolle sowie die krankheitsspezifische Letalität effektiv verbessert [1, 2]. Darüber hinaus zeigten die Ergebnisse der EORTC-1098122023-AMAROS-Studie, dass die axilläre Strahlentherapie nach positiver Sentinel-node-Biopsie den Patientinnen eine ausgezeichnete axilläre Kontrolle bietet, auch wenn auf die ALND verzichtet wird [3]. Aus den MA.20- und EORTC- 
22922-Studien geht jedoch auch hervor, dass die BCRLRate-basierend auf der subjektiven klinischen Beurteilung in MA.20 mit der RLNR anstieg, und zwar von 4,5\% auf $8,4 \%$ bzw. von $10,5 \%$ auf $12 \%$. Die AMAROS-Studie zeigte, dass auch Patienten mit 1-2 befallenen axillären Lymphknoten nach ALND (ohne RLNR) oder nach SLNB, gefolgt von RLNR, ähnliche Tumorkontrollraten erreichen können (5-Jahres-Gesamtüberleben 93,3\% bzw. 92,5\%; $p=0,34)$. Darüber hinaus betrug die BCRL-Inzidenz nach 5 Jahren in der SLNB+RLNR-Gruppe 6\%, im Vergleich zu $13 \%$ nach ALND allein. Diese Ergebnisse implizieren, dass das Risiko, ein BCRL zu entwickeln, mehr vom Ausmaß der axillären Operation als von der Bestrahlung abhängt.

Unverständlich ist uns, wieso in der Literatur nach ALND höhere Lymphödem-Raten berichtet werden (>20\% Risiko) als in der AMAROS-Studie [4-6]. Eine mögliche Erklärung wäre, dass in AMAROS für beide Gruppen eine Kombination von ALND und RLNR zugelassen war. Wenn $>4$ positive $\mathrm{LN}$ in einer Intention-to-treat-Analyse (ITT) vorhanden waren, kann das natürlich zu verzerrten BCRL-Raten führen, da ITT-Analysen den Effekt der Behandlungszuweisung schätzen und nicht den Behandlungseffekt. Eine andere Erklärung wäre die Methode, das Lymphödem zu quantifizieren. Welche Methode ist dafür überhaupt geeignet? Denn es ist ja bekannt, dass Umfangsänderungen der Gliedmaßen, gemessen mit einem Maßband, relativ ungenau sind und oftmals keine eindeutige Diagnose erlauben. Insbesondere können Schwellungen an verschiedenen Stellen des betroffenen Arms (z.B. an der Hand) dadurch nicht berücksichtigt werden. Andere Studien, wie z.B. IBCSG23-01, haben nach CTCAE nur eine Beurteilung des BCRL durch den Arzt erlaubt. Das bedeutete vermutlich mehr Subjektivität bei der BCRLDiagnose und könnte die unterschiedlichen Angaben in der Literatur erklären [7]. Bekannt ist auch, dass ein Perometer bei der Lymphödemeinschätzung zuverlässiger als eine Armumfangsmessung ist und auch deshalb oft als Screeningmethode empfohlen wird $[8,9]$.

Die in der hier kommentierten Studie beschriebenen prospektiven Methoden versuchen, die Fallstricke bei der Beurteilung von Lymphödemen zu umgehen. Darüber hinaus wird durch die Verwendung der RVC- oder WAC-Formel eine Kontrolle eingeführt, die Änderungen des Volumens des betroffenen Arms im Verhältnis zum kontralateralen Arm (RVC) oder zum Körpergewicht (WAC) beurteilt. Von ihnen ist bekannt, dass sie während und nach der Behandlung schwanken. Dadurch haben die Autoren der hier kommentierten Studie die Objektivität bei der Lymphödemeinschätzung erhöht und eine aussagekräftige Antwort auf die Frage der ,schuldigen“ Behandlungsmethode gegeben.

\section{Fazit}

Die hier diskutierte Studie konnte die unterschiedlichen BCRL-Raten in den verschiedenen Behandlungsgruppen sehr gut darstellen (,breast cancer-related lymphedema“" [BCRL]) mit SLNB allein (8\%), SLNB+RLNR $(10,7 \%)$, ALND (24,9\%) und ALND+RLNR (30,1\%). Obwohl die Patienten, die SLNB/ALND+RLNR erhielten, ein gering höheres Lymphödemrisiko hatten im Vergleich zu denen mit SLNB/ALND allein, war dieser Unterschied bei der multivariablen Analyse nicht signifikant. Der Hauptrisikofaktor für das BCRL bleibt also das Ausmaß der axillären Operation und nicht die zusätzliche RLNR. Das bestätigt die früheren AMAROS- und IBCSG23-01-Ergebnisse und unterstreicht den Nutzen der adjuvanten (regionalen Lymphknotenbestrahlung [RLNR]), die ohne eine zusätzliche signifikante Toxizität durchgeführt werden kann.

\section{Marciana-Nona Duma, Jena}

Funding Open Access funding enabled and organized by Projekt DEAL.

Interessenkonflikt M.-N. Duma gibt an, dass kein Interessenkonflikt besteht.

Open Access Dieser Artikel wird unter der Creative Commons Namensnennung 4.0 International Lizenz veröffentlicht, welche die Nutzung, Vervielfältigung, Bearbeitung, Verbreitung und Wiedergabe in jeglichem Medium und Format erlaubt, sofern Sie den/die ursprünglichen Autor(en) und die Quelle ordnungsgemäß nennen, einen Link zur Creative Commons Lizenz beifügen und angeben, ob Änderungen vorgenommen wurden.

Die in diesem Artikel enthaltenen Bilder und sonstiges Drittmaterial unterliegen ebenfalls der genannten Creative Commons Lizenz, sofern sich aus der Abbildungslegende nichts anderes ergibt. Sofern das betreffende Material nicht unter der genannten Creative Commons Lizenz steht und die betreffende Handlung nicht nach gesetzlichen Vorschriften erlaubt ist, ist für die oben aufgeführten Weiterverwendungen des Materials die Einwilligung des jeweiligen Rechteinhabers einzuholen.

Weitere Details zur Lizenz entnehmen Sie bitte der Lizenzinformation auf http://creativecommons.org/licenses/by/4.0/deed.de.

\section{Literatur}

1. Poortmans PM et al (2015) Internal mammary and medial supraclavicular irradiation in breast cancer. N Engl J Med 373(4):317-327

2. Whelan TJ et al (2015) Regional nodal irradiation in early-stage breast cancer. N Engl J Med 373(4):307-316

3. Donker $\mathrm{M}$ et al (2014) Radiotherapy or surgery of the axilla after a positive sentinel node in breast cancer (EORTC 10981-22023 AMAROS): a randomised, multicentre, open-label, phase 3 noninferiority trial. Lancet Oncol 15(12):1303-1310

4. Gillespie TC et al (2018) Breast cancer-related lymphedema: risk factors, precautionary measures, and treatments. Gland Surg 7(4):379-403

5. Giuliano AE et al (2017) Effect of axillary dissection vs no axillary dissection on 10-year overall survival among women with invasive breast cancer and sentinel node metastasis the ACOSOG Z0011 (alliance) randomized clinical trial. JAMA 318(10):918-926 
6. Liljegren G, Holmberg L (1997) Arm morbidity after sector resection and axillary dissection with or without postoperative radiotherapy in breast cancer stage. 1. Results from a randomised trial. Eur J Cancer 33(2): 193-199

7. Galimberti V et al (2013) Axillary dissection versus no axillary dissection in patients with sentinel-node micrometastases (IBCSG 23-01): a phase 3 randomised controlled trial. Lancet Oncol 14(4):297-305
8. Dylke ES, Ward LC, Kilbreath SL (2013) Standardized approach to lymphedema screening. Oncologist 18(11):1242

9. Ancukiewicz M et al (2011) Standardized method for quantification of developing lymphedema in patients treated for breast cancer. Int J Radiat Oncol Biol Phys 79(5):1436-1443 